\title{
Efectividad de los recursos y las aplicaciones tecnológicas utilizados por la población docente de la Escuela José Trinidad Mora Valverde, Costa Rica, en el periodo julio a setiembre de $\mathbf{2 0 2 0}$
}

\author{
Effectiveness of the resources and technological applications used by the \\ teaching population of the José Trinidad Mora Valverde School, Costa Rica in \\ the period July to September 2020
}

Eficácia dos recursos tecnológicos e aplicações utilizadas pelo corpo docente da Escola José Trinidad Mora Valverde, Costa Rica, no período de julho a setembro de 2020

\author{
Jacqueline Mora Fallas \\ Ministerio de Educación Pública \\ San José, Costa Rica \\ jaque88.jmf@gmail.com \\ (1) ORCID: https://orcid.org/ 0000-0001-5262-7786 \\ Ana Cristina Umaña Mata \\ Universidad Estatal a Distancia \\ San José, Costa Rica \\ acumanam@uned.ac.cr \\ (D) ORCID: https://orcid.org/0000-0002-7998-1061
}

Recibido - Received - Recebido: 19/03 / 2021 Corregido - Revised - Revisado: 08 / 08 / 2021 Aceptado - Accepted - Aprovado: 30 / 09 / 2021

DOl: https://doi.org/10.22458/ie.v23iespecial.3475

URL: https://revistas.uned.ac.cr/index.php/innovaciones/article/view/3475

\begin{abstract}
Resumen: El presente artículo contempla los principales resultados de la investigación, cuyo objetivo fue analizar la efectividad de los recursos y aplicaciones tecnológicas seleccionadas por la población docente de la Escuela José Trinidad Mora Valverde de Costa Rica, para el desarrollo de los procesos de mediación pedagógica en el periodo comprendido de julio a setiembre del 2020. El enfoque de investigación fue mixto; entre los principales resultados obtenidos, se destaca que los docentes han priorizado como recurso tecnológico el celular para los procesos de mediación pedagógica. Esto por cuanto, es el medio al que tienen mejor acceso y comunicación con los niños y los padres de familia. Además, se denota en este grupo de participantes, gran compromiso por generar espacios efectivos con el fin de favorecer la mediación pedagógica, con apoyo de las tecnologías. Sin embargo, se evidencia su poca experiencia en el uso de los recursos y las aplicaciones tecnológicas para fines educativos. Entre las conclusiones se destacan que los docentes están dando prioridad al uso de las aplicaciones tecnológicas Microsoft Teams ${ }^{\circledast}$ y WhatsApp ${ }^{\circledR}$ con fines educativos, para la estrategia "Aprendo en casa" implementado por el Ministerio de Educación Pública, ya que estas dos aplicaciones son las más utilizadas por los docentes. Además es clara la necesidad de fortalecer las capacitaciones para el mejor uso de las diversas aplicaciones tecnológicas con que cuentan para los procesos de enseñanza y aprendizaje.
\end{abstract}

Palabras claves: Educación a distancia, tecnologías de la información, papel docente, pandemia, investigación.

Summary: This article contemplates the main results of the research, the purpose of which was to analyze the effectiveness of the resources and technological applications selected by the teaching population of the José Trinidad Mora Valverde School of Costa Rica, for the development of the pedagogical mediation processes in the period from July to September 2020. The research approach was mixed. Among the main results obtained, it stands out that teachers have prioritized cell phones as a technological resource for pedagogical mediation processes. This inasmuch as it is the means to which they have the best access and through which they have the best communication with children and parents. In addition, this group of participants shows a great commitment 
to generate effective spaces in order to favor pedagogical mediation, with the support of technologies. However, their little experience in the use of resources and technological applications for educational purposes is evident. Among the conclusions, it is highlighted that teachers are giving priority to the use of Microsoft Teams ${ }^{\circledR}$ and WhatsApp ${ }^{\oplus}$ technological applications for educational purposes, and for the "I learn at home" strategy implemented by the Ministry of Public Education, since these two applications are the most used by teachers. In addition, the need to strengthen training for the best use of the various technological applications available for teaching and learning processes is clear.

Key Words: Distance education, information technology, teaching role, pandemic, research

Resumo: Este artigo inclui os principais resultados da pesquisa, cujo objetivo foi analisar a eficácia dos recursos tecnológicos e aplicações selecionadas pela população docente da Escola José Trinidad Mora Valverde na Costa Rica, para o desenvolvimento dos processos de mediação pedagógica no período de julho a setembro de 2020. A abordagem da pesquisa foi mista; entre os principais resultados obtidos, destaca-se que os professores priorizaram o telefone celular como recurso tecnológico para os processos de mediação pedagógica. Isto porque é o meio ao qual eles têm o melhor acesso e comunicação com as crianças e os pais. Além disso, este grupo de participantes está altamente comprometido em gerar espaços efetivos para favorecer a mediação pedagógica com o apoio da tecnologia. Entretanto, sua falta de experiência no uso de recursos tecnológicos e aplicações para fins educacionais é evidente. Entre as conclusões, os professores estão priorizando o uso das aplicações tecnológicas Microsoft Teams ${ }^{\circledast}$ e WhatsApp ${ }^{\circledR}$ para fins educacionais, para a estratégia "Eu aprendo em casa" implementada pelo Ministério da Educação Pública, já que estas duas aplicações são as mais utilizadas pelos professores. Além disso, há uma clara necessidade de reforçar o treinamento para o melhor uso das diversas aplicações tecnológicas para os processos de ensino e aprendizagem.

Palavras-chave: Educação à distância, tecnologias da informação, papel do professor, pandemia, pesquisa.

\section{INTRODUCCIÓN}

En el 2019, por motivo de una huelga del profesorado del Ministerio de Educación Pública de Costa Rica (MEP), el curso lectivo fue suspendido y finalizado abruptamente, sin que se cumplieran los objetivos en cada uno de los niveles. Esta situación obligó a tomar una serie de previsiones para el 2020, con el fin de considerar los posibles faltantes en el desarrollo de conocimientos por parte del estudiantado (Programa del Estado de la Nación, 2019). Dicho informe también reveló que, a nivel nacional, se identifican serios problemas de cobertura y calidad en cuanto a la oferta educativa en primaria y secundaria.

Aunado a lo anterior, para el 2020, se dispuso la aplicación de un nuevo modelo de evaluación de los aprendizajes, el cual está orientado por criterios y acumulación de evidencias en el proceso del curso lectivo. El panorama descrito en los párrafos anteriores, deja claro el reto que debía enfrentar el sistema educativo costarricense para el 2020, con el fin de subsanar los vacíos ya existentes. Sin embargo, lo que no se podría haber considerado era la crisis mundial por la pandemia que afectó al país en su totalidad, a causa el virus responsable de la COVID-19. Este evento, dejó paralizadas gran cantidad de actividades de Costa Rica, incluidas aquellas que involucraban el desarrollo habitual del curso lectivo, dadas las medidas implementadas por el Ministerio de Salud, entre las cuales el distanciamiento social ha sido la constante.

Ante la pandemia y, con los antecedentes de la situación suscitada en el 2019, se toma como medida de emergencia que el personal docente implemente clases con uso de tecnologías, sin que se pudiera desarrollar la capacitación correspondiente de manera profunda, eficiente y oportuna (Programa del Estado de la Nación, 2019).

A la fecha, hay poca evidencia del desarrollado de investigaciones para determinar las aplicaciones y los recursos tecnológicos seleccionados por la población docente para el desarrollo de la mediación pedagógica ante la pandemia. Por ello, el estudio realizado, tuvo como objetivo general analizar a través de triangulación de datos los alcances de la utilidad educativa de los recursos y las aplicaciones tecnológicas seleccionadas por la población docente de la Escuela José Trinidad Mora Valverde, para el desarrollo de los procesos de mediación pedagógica en el periodo comprendido de julio a setiembre de 2020. Para el logro de dicho objetivo, se determinaron los siguientes objetivos específicos: 
- Identificar los recursos y las aplicaciones tecnológicas seleccionados por la población docentes de la Escuela José Trinidad Mora Valverde para el desarrollo de los procesos de mediación pedagógica en el periodo comprendido de julio a setiembre de 2020.

- Determinar por medio de grupos focales los fines educativos de los recursos y las tecnológicas seleccionados, según su intencionalidad de creación.

- Establecer por medio de la aplicación de una encuesta la efectividad educativa en el uso de los recursos y las aplicaciones tecnológicas seleccionadas por los docentes obtenida en el momento de implementarlas.

En cuanto a los referentes teóricos de esta investigación, se parte por comprender la mediación pedagógica como el proceso en el que se lleva a cabo una interacción entre la persona docente y el estudiantado:

[...] en el cual se orientan las diferentes acciones educativas para la consecución de la transformación o reestructuración de los procesos de aprendizaje en cada estudiante, de manera que permita realizar un enlace entre cada estudiante y el objeto de conocimiento. (UNED, 2013, p. 41).

Por lo tanto, se parte de la premisa de que este tipo de espacio tiene como fin propiciar los espacios necesarios, para la generación de conocimientos que promuevan el aprendizaje en el estudiantado.

Además, es necesario tener en cuenta que la mediación pedagógica, se ejerce por diversos medios y recursos y el cuerpo docente tiene la responsabilidad del desarrollo de tal función, lo cual implica un cambio en su rol. Al respecto, Viñals y Cuenca (2016) refieren que, en este momento la forma de aprender ha cambiado dando paso al proceso de aprender a aprender" donde la figura del docente aparece con un papel protagónico en los procesos de generación de metodologías alternativas, para que el estudiantado pueda favorecer el desarrollo de estrategias en el estudiantado, de manera que puedan acceder a formas diversas de aprender.

Por lo tanto, se puede indicar que el profesor será el mediador en este proceso de enseñanza-aprendizaje y siempre debe estar preparado, no solo en su especialidad, sino también en el uso de tecnologías en el proceso educativo. Hoy se sabe que, muchos docentes muestran resistencia al uso de las nuevas tecnologías, esto porque no sienten tener los conocimientos necesarios para este nuevo reto, u otro caso porque no cuentan con los recursos necesarios.

Los recursos y las aplicaciones tecnológicas forman parte de las tecnologías de información y comunicación (TIC). En el caso de los recursos se utilizan para gestionar, procesar, almacenar y presentar información, los cuales se han convertido en instrumentos que facilitan muchas de las labores que desarrollan los seres humanos en la actualidad, esto se ve reflejado en la función que cumplen en la mediación de los procesos de enseñanza-aprendizaje. (Hernández, Gómez y Balderas 2014)

Por otra parte, los recursos tecnológicos pueden definirse en tangibles e intangibles. Los recursos tecnológicos tangibles son los que pueden ser físicamente manipulados por la persona usuaria. En esta clasificación se incluyen la computadora, la impresora, el celular, el iPad o tableta, las pizarras electrónicas. Por su parte, los recursos tecnológicos intangibles, tal como su nombre lo indica, no son visibles ni manipulables directamente por los usuarios, en este caso se incluyen los sistemas de software que se descargan para diversos fines, pueden incluirse acá, las aplicaciones como Microsoft Teams', Zoom, WhatsApp', Facebook', Google Classroom ', entre otras.

La aplicación WhatsApp muy utilizada a nivel mundial, tanto por personas adultas como personas menores de edad y, por supuesto, la población estudiantil no es la excepción al igual que los docentes; es de gran utilidad para la comunicación tanto escrita como por audio. Según Acuña (2017, s.f.) el WhatsApp "[...] es una de las mensajerías más utilizadas en el mundo y aunque conocemos los problemas de 
seguridad y las veces que han colapsado sus servidores, imposible dejar de usarlo". Hoy esta aplicación es muy importante para la comunicación virtual entre nosotros los docentes y entre nuestros estudiantes; ya que nos permite la creación de grupos en los cuales participan gran cantidad de personas.

WhatsApp puede tener fines motivadores y tiene grandes posibilidades para favorecer la comunicación de diversos tipos; pues ofrece únicamente mensajes escritos, mensajes de voz, imágenes, fotografías, video llamadas. También es importante porque permite el desarrollo de la comunicación y expresión escrita; busca que los estudiantes desarrollen o exploten sus habilidades de redacción para expresar sus ideas, y no solo escrito sino también por medio del audio.

Otra aplicación muy utilizada tanto por los docentes como por los estudiantes es Facebook . Al igual que el WhatsApp', la gran mayoría tiene acceso a esta, razón por la cual se le podrá sacar gran provecho en actividades propias del proceso educativo. Al respecto, Palmucci (2015) plantea que, se genera un tipo de vínculo diferente entre el personal docente y el estudiantado, así como un cambio en la concepción del aula, la cual deja de ser un espacio cerrado, para convertirse en un espacio de diálogo e intercambios de conocimiento, que puede dar posibilidades a los espacios de aprendizaje colaborativo. Por su parte, Zoom es una aplicación que previo a la pandemia no era muy conocida por todos. Es fácil de utilizar y sirve para el desarrollo de clases de manera sincrónica; pues como aplicación, permite el desarrollo de videoconferencias basada en la nube que integra una serie de funcionalidades, tales como: audio, vídeo, chat, compartir contenido, además de otras formas de interactuar con los participantes. Entre sus opciones, se puedan grabar las sesiones para luego compartirlas y permite crear salas virtuales para trabajo en grupo.

Por su parte, Microsoft Teams es una aplicación que antes de que se diera la pandemia producto del virus por la COVID-19 y se iniciaran las clases virtuales planteadas por el Ministerio de Educación Pública de Costa Rica (MEP) muy pocas personas la conocían. Sin embargo, en la actualidad, muchos docentes tanto de primaria como de secundaria la utilizan en el desarrollo de sus lecciones por normativa del MEP.

Esta aplicación tiene diferentes usos, los cuales se pueden utilizar de la manera que más le funcione a la persona docente. Con esta aplicación se puede realizar las siguientes actividades, según lo indica Mazas (2018):

a) Equipos: busque los canales de los que quiere formar parte o cree uno. Dentro de los canales, puede organizar reuniones, mantener conversaciones y compartir archivos.

b) Reuniones: compruebe que tiene listo para el día o la semana. O bien, programe una reunión, por medio del calendario.

c) Llamadas: en algunos casos, si su organización lo ha configurado, puede llamar a quien desee Teams, incluso si esa persona no lo está usando.

A manera de síntesis, en la tabla 1 se presentan las aplicaciones tecnológicas según sus posibles usos educativos:

TABLA 1

Aplicaciones tecnológicas según sus posibles usos educativos

\begin{tabular}{ccccccc} 
Aplicación & $\begin{array}{c}\text { Brindar } \\
\text { información }\end{array}$ & $\begin{array}{c}\text { Envío de } \\
\text { materiales }\end{array}$ & $\begin{array}{c}\text { Desarrollo de espacios } \\
\text { de interacción }\end{array}$ & $\begin{array}{c}\text { Trabajo } \\
\text { colaborativo }\end{array}$ & Presentaciones & Motivación \\
\hline WHATSAPP $^{\circ}$ & $\mathrm{X}$ & $\mathrm{X}$ & & $\mathrm{X}$ & $\mathrm{X}$ & $\mathrm{X}$ \\
\hline $\mathrm{ZOOM}^{\circ}$ & $\mathrm{X}$ & & $\mathrm{X}$ & $\mathrm{X}$ & $\mathrm{X}$ & $\mathrm{X}$ \\
\hline FACEBOOK $^{\circ}$ & $\mathrm{X}$ & $\mathrm{X}$ & & & & \\
\hline MICROSOFT TEAMS $^{\circ}$ & $\mathrm{X}$ & & $\mathrm{X}$ & $\mathrm{X}$ & $\mathrm{X}$ & $\mathrm{X}$ \\
\hline
\end{tabular}

Fuente: Elaboración propia. 
En relación con la tabla, se concluye que las aplicaciones consideradas en este estudio tienen finalidades de brindar información. A excepción de Facebook, las demás aplicaciones tecnológicas pueden utilizarse para el desarrollo de espacios interactivos, el desarrollo de trabajos colaborativos y la motivación para el desarrollo de los aprendizajes.

Concretamente en el campo de la educación, con la aparición de las TIC, se genera una serie de cambios, en diversos ámbitos del desarrollo de las personas; pues se requiere de la búsqueda de opciones que, permitan la incorporación de las tecnologías, en los procesos educativos. Al respecto, Marqués (2013, citado por Martínez-Garrido, 2018) indica que:

Las escuelas pueden optar por realizar pequeños ajustes en el currículum para que se utilicen los recursos tecnológicos como fuente de información (escenario tecnócrata), pueden además introducir este tipo de recursos en la metodología docente (escenario reformista) o pueden llegar a reestructurar los elementos de la escuela de manera global haciendo que la actividad de toda la escuela cambie (escenario holístico). (p.139)

Por lo tanto, en la actualidad, dejar de lado la existencia de la tecnología como derrotero en el desarrollo de un proceso de aprendizaje, parece ser una tarea impensable. Tal como lo indican Hernández, Gómez y Balderas (2014), las TIC y propiamente los recursos y las aplicaciones tecnológicas brindan gran cantidad de ventajas y opciones para su utilización en los contextos educativos. Esto por cuanto, al ser utilizadas, el personal docente puede generar nuevas y variadas formas de acercarse al conocimiento.

Para Cordero, De Roux, Jiany, Wong, Cattafi (2018, p. 35), todos los recursos tecnológicos que se utilizan a manera de medios para el desarrollo y el mejoramiento de los procesos de aprendizaje, se denominan recursos tecnológicos didácticos (RTD). Según los autores, puede decirse que un RTD es “[...] todo aquel medio material o conceptual que se utiliza como apoyo en la enseñanza [...] con la finalidad de facilitar o estimular el aprendizaje". (p.35)

Finalmente, es necesario identificar las principales funciones que podrían tener los recursos y aplicaciones tecnológicas, cuando se utilizan para el desarrollo de procesos educativos. Al respecto, la Asociación de educación abierta, (2018, párrafo 4) los resume en los siguientes puntos:

- Permite evaluar los conocimientos de los alumnos en cada momento, ya que normalmente suelen contener una serie de cuestiones con el fin de que el alumno reflexione.

- Facilitar el aprendizaje del alumno proporcionándoles información.

- Son una guía para los aprendizajes, ya que ayudan al docente en la organización de la información que éste desea transmitir.

- Ayuda a ejercitar y desarrollar las habilidades, ya que proporcionan un entorno para la expresión del alumno, ya se completando una ficha mediante una conversación en la que alumno y docente interactúan, ya sea a través de juegos de rol.

- Despiertan la motivación, la impulsan y crean un interés hacia el contenido mismo.

Con respecto al tema de la efectividad educativa, según Muñoz-Quezada (2006), debe evaluarse a partir de los aprendizajes significativos que logre la población estudiantil. Por ello, la autora considera que resulta fundamental el desarrollo de una mediación pedagógica que integre; además de los recursos, los medios y los materiales, el contexto y el sistema educativo como tal, caracterizado por una serie de principios y lineamientos que deberían estar orientados a la calidad, eficacia y equidad. 
La investigación realizada se circunscribió dentro del enfoque mixto; pues el cumplimiento de los objetivos específicos planteados responden en unos casos a la búsqueda de datos cualitativos $y$, en otros, de tipo cuantitativo. El primero objetivo específico se desarrolló mediante la implementación de la técnica de encuesta, lo que permitió la recolección de datos cuantitativos. En el caso del segundo objetivo específico, se orientó a la recolección de percepciones y opiniones de los participantes en el estudio. Por lo tanto, su orientación es claramente cualitativa y será atendido, mediante el desarrollo de grupos focales. Por último, el tercer objetivo que está enfocado en un cuestionario que brindará datos cuantitativos. En cuanto al diseño de la investigación considera la inclusión del diseño cuantitativo cuyo nivel de alcance será exploratorio y del diseño cualitativo de tipo narrativo.

En cuanto a la población que se involucra en esta investigación está compuesta por todos los docentes de la Escuela José Trinidad Mora Valverde. En esta investigación participaron los docentes de primer y segundo ciclo de la Educación General Básica que impartía las materias generales: español, ciencias, estudios sociales y matemáticas. Para un total de población de 27 personas encuestadas.

Como datos generales de la población participante, la mayoría de los docentes encuestados cuentan con grado de licenciatura (21 en total). Con grado de bachillerato universitario hay cuatro personas y solamente dos tienen maestría. El nivel de escolaridad es muy importante para determinar la formación y la actualización de la población docente. Los datos obtenidos refieren que, por el grado de escolaridad, es una población con la suficiente preparación académica para atender, de la manera más adecuada, la mediación pedagógica en sus procesos de enseñanza.

Según la información recolecta en el cuestionario el grupo de personas docentes que llenaron el cuestionario es bastante heterogéneo, en cuanto a los años de experiencia laboral en la institución. En el rango de uno a cinco años se ubica la mitad de la población encuestada y, el resto tiene más de seis años de laborar en la escuela. La permanencia en un lugar de trabajo, además de la estabilidad laboral que brinda a las personas, les ofrece la oportunidad de generar una identidad institucional y, en este caso en particular, cobra mucha relevancia el tiempo de permanencia del persona docente; pues con ello logran un conocimiento amplido de la comunidad en donde está el centro educativo, así como de las condiciones socioeconómicas de la población estudiantil.

En relación con el género del personal docente, la mayoría son femeninas lo cual corresponde un 96,3\% y uno es masculino, (3,7\%). En cuanto a la edad de las personas, nueve de ellos se encuentran entre 36 a 45 años, por lo que representa el 33,3\%; siete de ellos se ubican entre 46 a 55 años que representa el $25,9 \%$, seis de ellos se encuentran entre 25 a 35 años que representa el $22,2 \%$, cuatro se hallan entre 55 años y más que representa el $14,8 \%$ y un participante se ubica entre menos de 25 años que representa el 3,7\%. Los datos obtenidos indican que, hay un grupo importante de personas que no pertenecen a la llamada era digital; es decir, su formación y el desarrollo de gran parte de su vida no contó con la tecnología como un componente vital.

Para la realización del estudio, se definieron tres variables de investigación. La primera de ellas fue "Recursos y aplicaciones tecnológicas", cuya definición conceptual considera los recursos y las aplicaciones tecnológicas como medios que utilizan la tecnología para cumplir sus propósitos. Dicha variable se estudió mediante el instrumento de encuesta y corresponde al objetivo número uno de la investigación. La segunda variable es "La efectividad educativa en el uso de recursos y aplicaciones tecnológicas", entendida como la relación de coherencia entre los objetivos educativos, los cuales se establecen previamente y, los resultados obtenidos a partir del uso de los recursos y las aplicaciones tecnológicas en procesos de aprendizaje. Esta segunda variable se midió por medio de una escala de Likert y, abarcaba el cumplimiento del segundo objetivo de la investigación. 
Una tercera variable, directamente relacionada con el objetivo número tres, fue "La mediación pedagógica". Para los efectos de la investigación, se conceptualizó como el proceso de interacción mediante el cual la persona docente orienta las acciones educativas, con el fin de transformar o reconstruir los procesos de aprendizaje del estudiantado. En este caso se trabajó con un grupo focal.

En cuanto a las fases del procedimiento de recolección de datos, estas se desarrollaron en cuatro fases predeterminadas para la recolección de la información. Esas etapas se ejecutaron de forma secuencial, tal y como se describen a continuación. Esta planificación, buscó garantizar que el proceso fuera efectivo y permitiera obtener los datos requeridos.

La primera fase del proceso inició con la exhaustiva revisión de literatura sobre temas afines a los de interés de las personas investigadoras. En esta fase, interesaba valorar la pertinencia de seleccionar el tema de investigación, así como la definición de las variables de investigación que serían consideradas. En una segunda fase, se llevó a cabo la selección de las técnicas y el diseño de los instrumentos para recolectar los datos. En este proceso, resultó fundamental recurrir a la revisión de los objetivos de la investigación, de manera que se lograra identificar si el cumplimiento de cada uno de ellos implicaba el uso de técnicas cualitativas o cuantitativas.

Como tercera fase, se procedió a efectuar el primer contacto con los participantes previamente seleccionados. Este acercamiento fue vía correo electrónico y, se presentó a las posibles personas participantes, el detalle del propósito y los objetivos del estudio mediante una carta, en la cual se indica el respeto por su identidad y se mantendrá en el anonimato, lo mismo que las posibilidades que tiene de retirarse y declinar su participación en cualquier momento. De igual manera, se les remitió el consentimiento informado para contar con la aceptación de las personas participantes en el estudio.

La cuarta fase consistió en el análisis de los datos. Para tal efecto, se consideró un programa que permite el procesamiento de la información de la encuesta denominado Google Forms. Por otra parte, se transcribió la información recolectada en el grupo focal. Posteriormente, se clasificó según las unidades de análisis que fueron determinadas de acuerdo con la información recolectada y, las posibles vinculaciones para el logro de los objetivos de la investigación.

\section{DISCUSIÓN DE RESULTADOS}

Con respecto al cumplimiento del objetivo número uno: identificar por medio de una encuesta los recursos y las aplicaciones tecnológicas seleccionados por la población docentes de la Escuela José Trinidad Mora Valverde para el desarrollo de los procesos de mediación pedagógica en el periodo comprendido de julio a octubre de 2020. Los datos obtenidos son contundentes en que el medio más utilizado es el celular y la Tablet el que menos se usa. Al respecto, se puede interpretar que, la selección del recurso está vinculada con las posibilidades de que, sea un recurso compatible para trabajar con los niños, y posiblemente de fácil acceso.

Una de las variables que interesaba medir en esta investigación se vincula con las aplicaciones tecnológicas de preferencia por el personal docente y, los fines para los cuales las utilizaban en el trabajo de mediación pedagógica. En ese sentido, tal como se presenta en la figura número uno, el WhatsApp es la aplicación más seleccionada para fines educativos, en especial para enviar información y documentos, lo mismo que para generar algún tipo de interacción. Llama la atención que, un grupo de ocho docentes la utiliza para el desarrollo colaborativo, lo cual resulta ser una tarea muy compleja por las características de la aplicación. En ese sentido, se rescata el esfuerzo creativo de este grupo de profesionales. 
Figura 1. Utilidad del WhatsApp ${ }^{\oplus}$ en procesos de enseñanza y aprendizaje

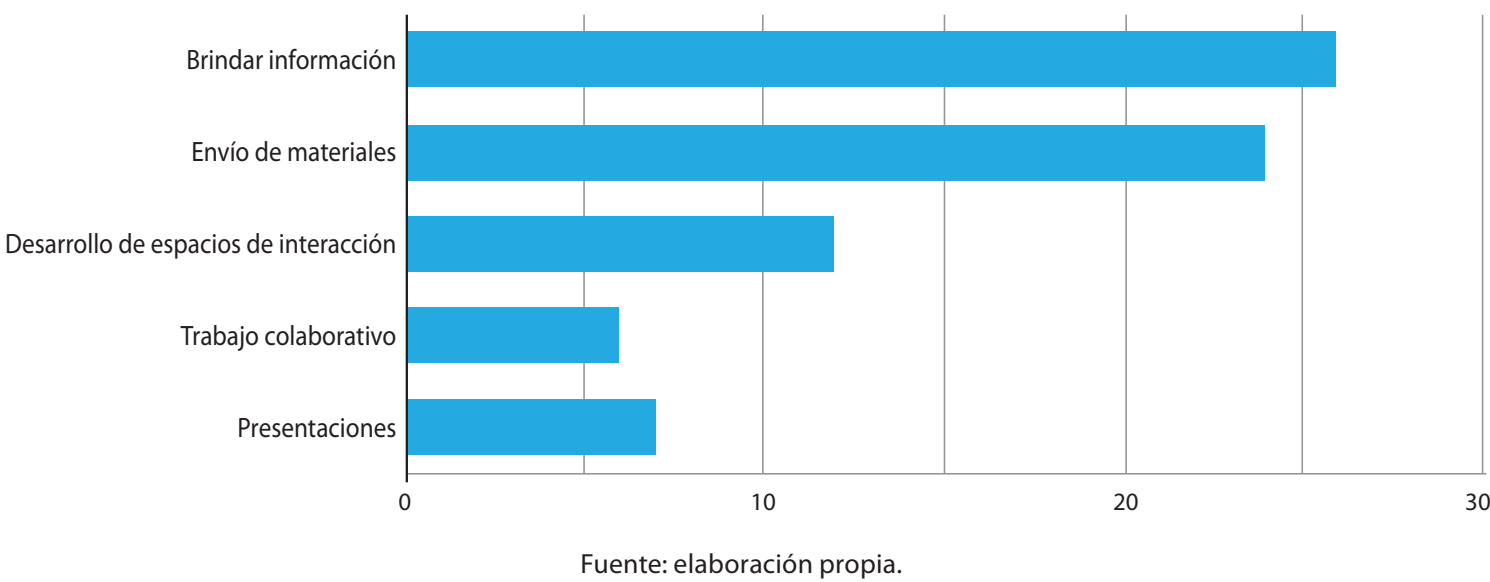

En cuanto a su utilidad, se confirma lo indicado por Acuña (2017) con respecto al uso de WhatsApp', quien indicó que esta aplicación es la de mayor utilidad a nivel mundial, pero también permite promover una mejor comunicación e interacción entre sus usuarios.En este caso en particicular con fines educativos.

Otra de las aplicaciones tecnológicas por las que se le consultó al personal docente fue sobre Facebook y su aplicación para los procesos de enseñanza y aprendizaje. En este caso, la respuesta se orientó a que su usó en mayor medida para enviar información. Esta aplicación tiene limitaciones importantes para el envío de documentos, pues en sí misma es una red social, de manera que se intuye que está siendo utilizada para ofrecer información o instrucciones, no tanto para envío de documento.

En las respuestas de las personas encuestadas, se nota que once de ellas trabajan en este espacio para motivación de los estudiantes, lo cual es muy acertado; pues da las alternativas para ello. Sin embargo, los estudios refieren que esta red es utilizada por persona adultas mayores de 30 años, dado que para la juventud es poco atractiva.

En la figura número dos, con respecto a la utilidad que los docentes le dan a Microsoft Teams, para algunas actividades educativas como parte de los procesos de mediación pedagógica, se encontró que la mayoría de ellos la utilizan para brindar información, envío de materiales y el desarrollo de espacios interactivos entre el estudiantado. Esta aplicación se usa también como medio para el desarrollo de trabajos colaborativos y la realización de presentaciones por parte del personal docente. En este caso en particular, varias de las funciones más importantes de la aplicación, como son aquellas relacionadas con los espacios de interacción y comunicación sincrónica, no se utilizan. El incremento en las respuestas por el uso de esta aplicación se asocia como producto a la normativa emitida por el Ministerio de Educación Pública, desde donde se ha dado la instrucción de que Microsoft Teams sea el medio oficial de comunicación con la población estudiantil. 
Figura 2. Utilidad de Microsoft Teams ${ }^{\circledast}$ en procesos de enseñanza y aprendizaje

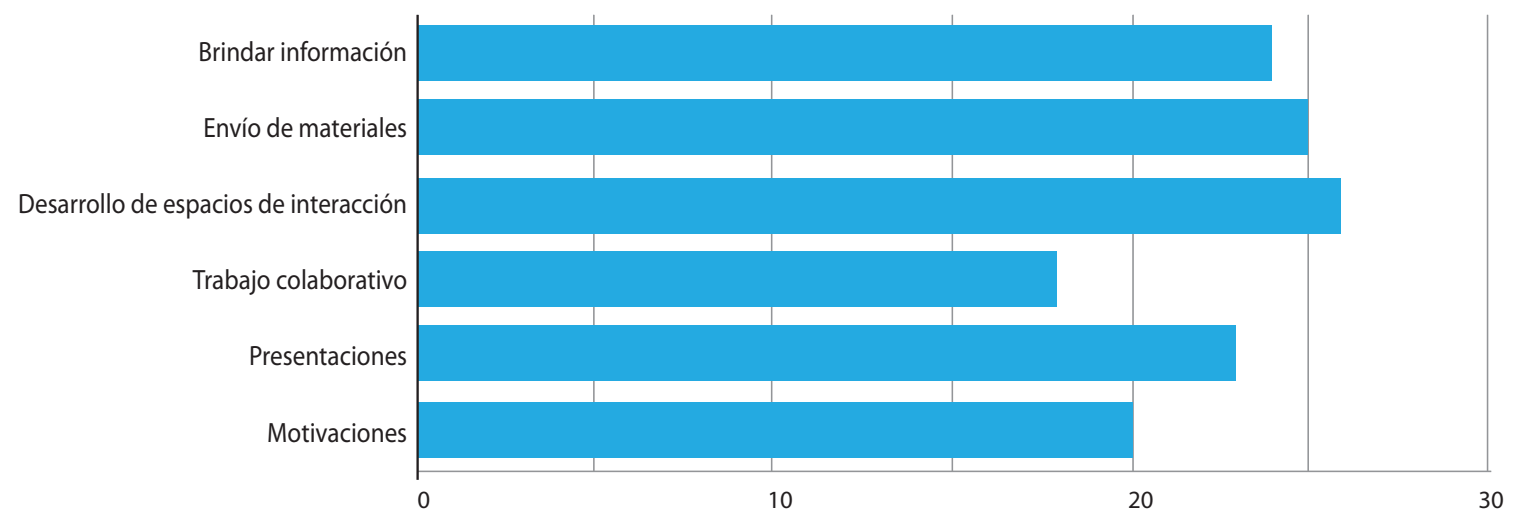

Fuente: elaboración propia.

Otra de las aplicaciones por las cuales se hizo consulta sobre su utilización en lo procesos de mediación pedagógica fue Zoom: Según los datos obtenidos, el 100\% de los encuestados refieren a que es un medio de interactuar con la población estudiantil, lo cual resulta coherente con una de sus principales funciones. Sin embargo, llama la atención que ocho de los docentes la utilizan para el envío de información, a pesar de ser un medio que puede usarse particularmente complejo para esta tarea tal como se muestra en la figura tres.

Figura 3. Utilidad de Zoom para el desarrollo de procesos de enseñanza y aprendizaje

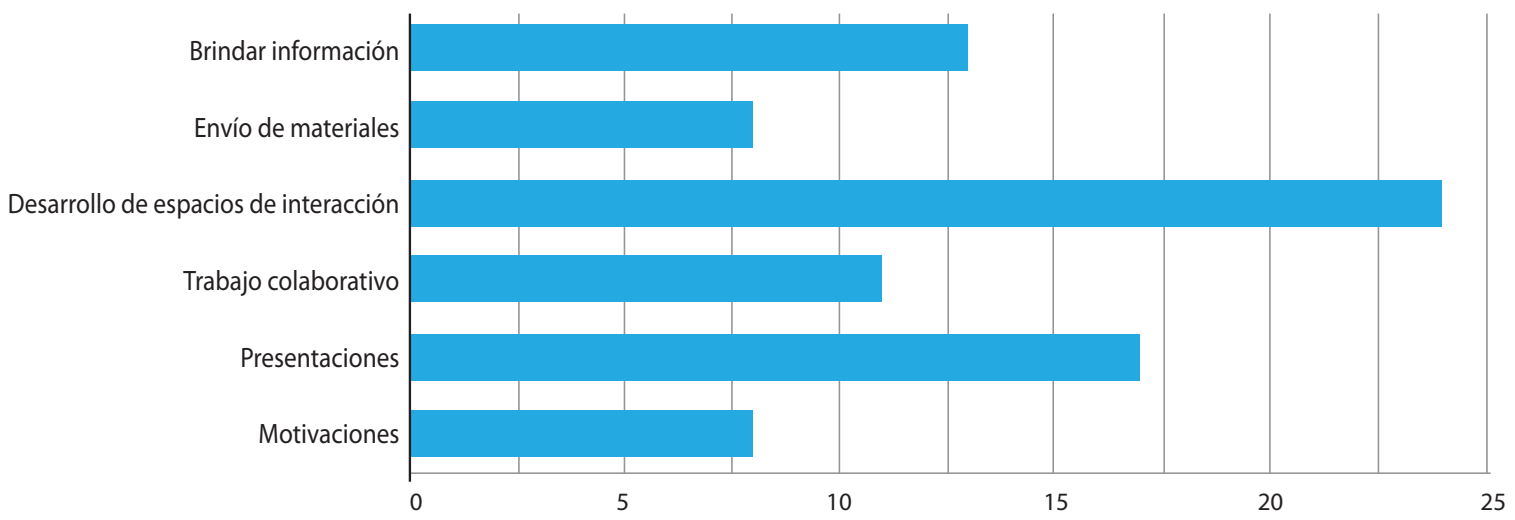

Fuente: elaboración propia.

Por otra parte, para utilizar Zoom 'se requiere contar con una licencia o el desarrollo de sesiones que no superen los 40 minutos. Desde el punto de vista pedagógico, se puede interpretar que, el uso de esta aplicación resulta funcional para impartir lecciones más orientadas hacia modelos magistrales y con un periodo previamente planificado.

Con respecto al objetivo número dos: establecer por medio de la aplicación de una encuesta y escala de Likert la efectividad educativa en el uso de los recursos y las aplicaciones tecnológicas seleccionadas por los docentes obtenida en el momento de aplicación del instrumento. En primera instancia se pidió la valoración de los criterios considerados por ellos para la selección de los recursos tecnológicos. En la figura número cuatro se recopilan los resultados con respecto a si la selección del recurso tecnológico ha facilitado el desarrollo del proceso de mediación pedagógica, en cuanto a la comunicación clara y precisa con la población estudiantil fue un criterio considerado por ellos. Al respecto, se encuentra que 
once de ellos están de acuerdo, el resto de las personas encuestadas tienen posiciones que dejan manifiesto que no fue un criterio a considerar. Se puede interpretar que, en términos generales, menos de la mitad de los encuestados consideró la facilidad de comunicación como un criterio, a pesar de que todos seleccionaron celulares, recurso específicamente para los fines indicados en este caso.

Figura 4. Facilidad de la comunicación clara y precisa con la población estudiantil por medio de recursos tecnológicos

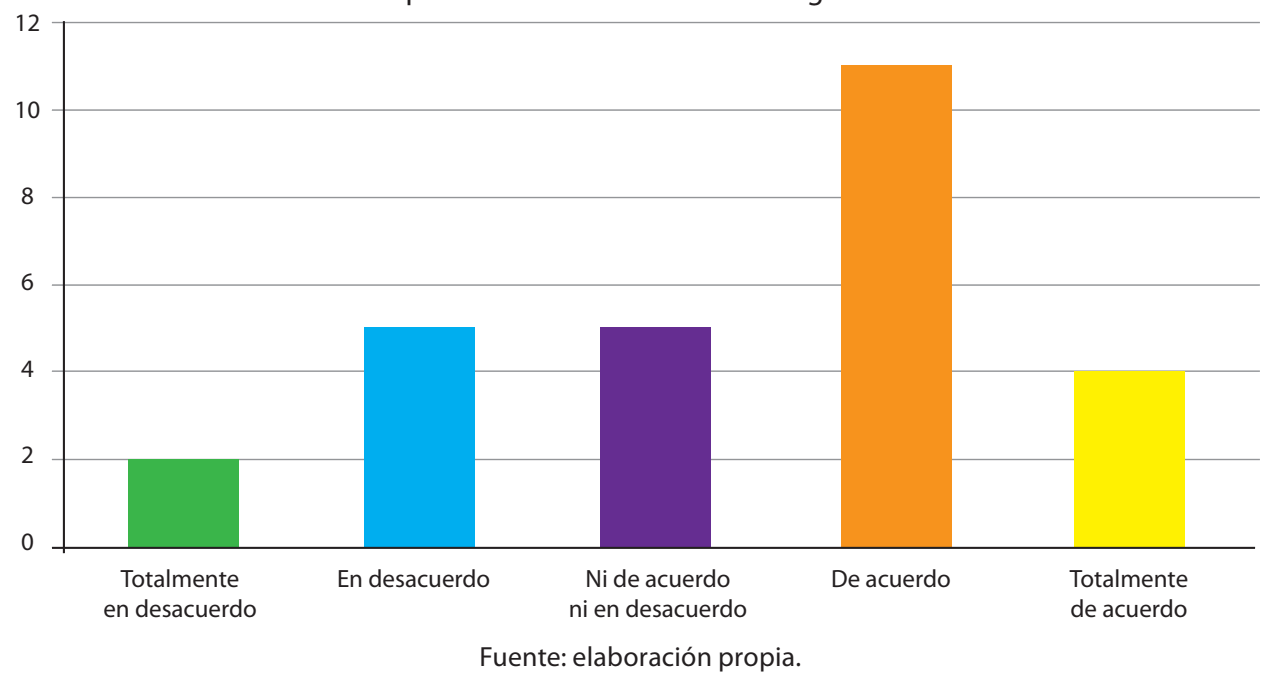

Otro de los criterios que se consultó para determinar la efectividad del recurso seleccionado, fue la facilidad para la presentación de los contenidos de los materiales para el proceso de aprendizaje del estudiantado. En este caso, más de la mitad del personal docente encuestado indica que sí se lograba. Por tanto, se interpreta que, dado el modelo presencial de impartir las lecciones, el uso de recursos que permitan de alguna manera mantener ese tipo de prácticas, sería considerado positivamente por el personal docente de la Escuela José Trinidad Mora Valverde, tal como se puede observar en la figura número cinco.

Figura 5. Facilidad de los recursos tecnológicos para la presentación de los contenidos para el desarrollo de los procesos de mediación pedagógica

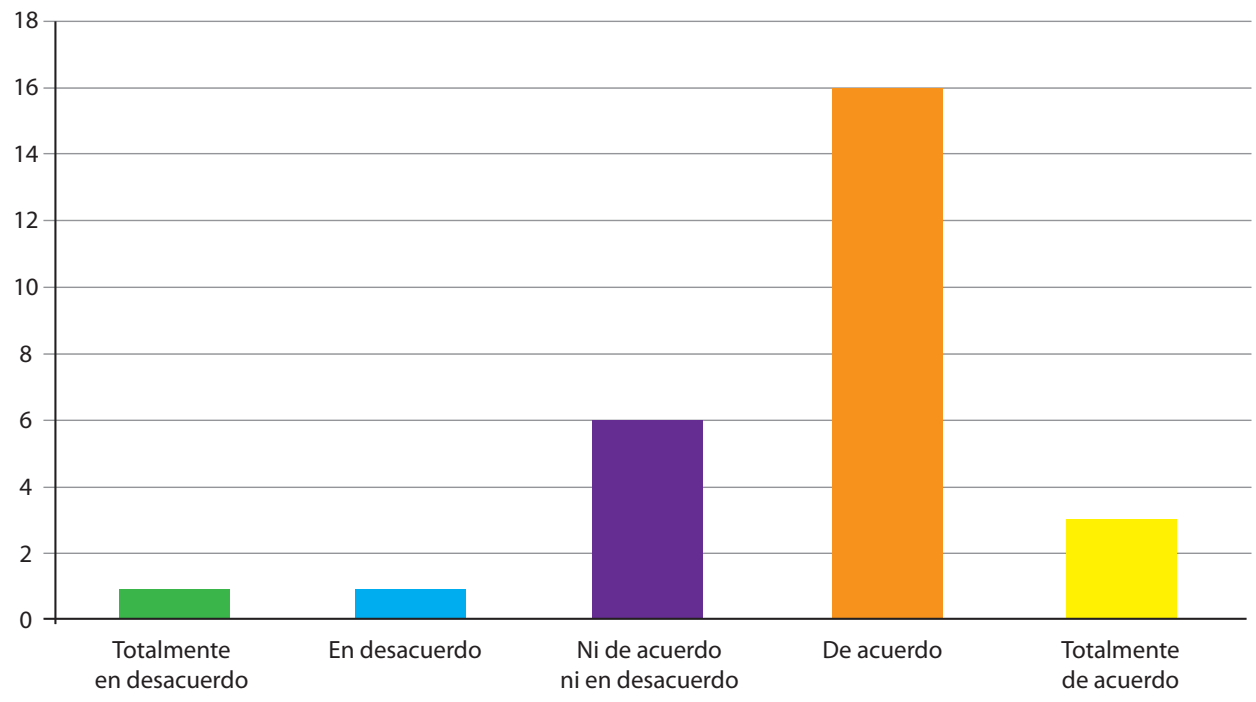

Fuente: elaboración propia. 
Tal como se puede visualizar en la figura número seis, relacionada con el tema de las facilidades para el desarrollo de la mediación pedagógica con el uso de los recursos tecnológicos, con respecto a la comunicación de forma clara y precisa las instrucciones del trabajo a desarrollar por parte del estudiantado, por parte del personal docente de la Escuela José Trinidad Mora Valverde. Puntualmente, más de la mitad de las personas encuestadas está de acuerdo con la efectividad de los recursos seleccionados para la comunicación precisa de instrucciones. Este dato es coincidente con lo que manifestaban respecto a los principales usos de las aplicaciones tecnológicas, la transmisión de información es de mayor peso que otro tipo de actividades.

Figura 6. Opinión sobre las facilidades para comunicar instrucciones al estudiantado, mediante el uso de recursos tecnológicos.

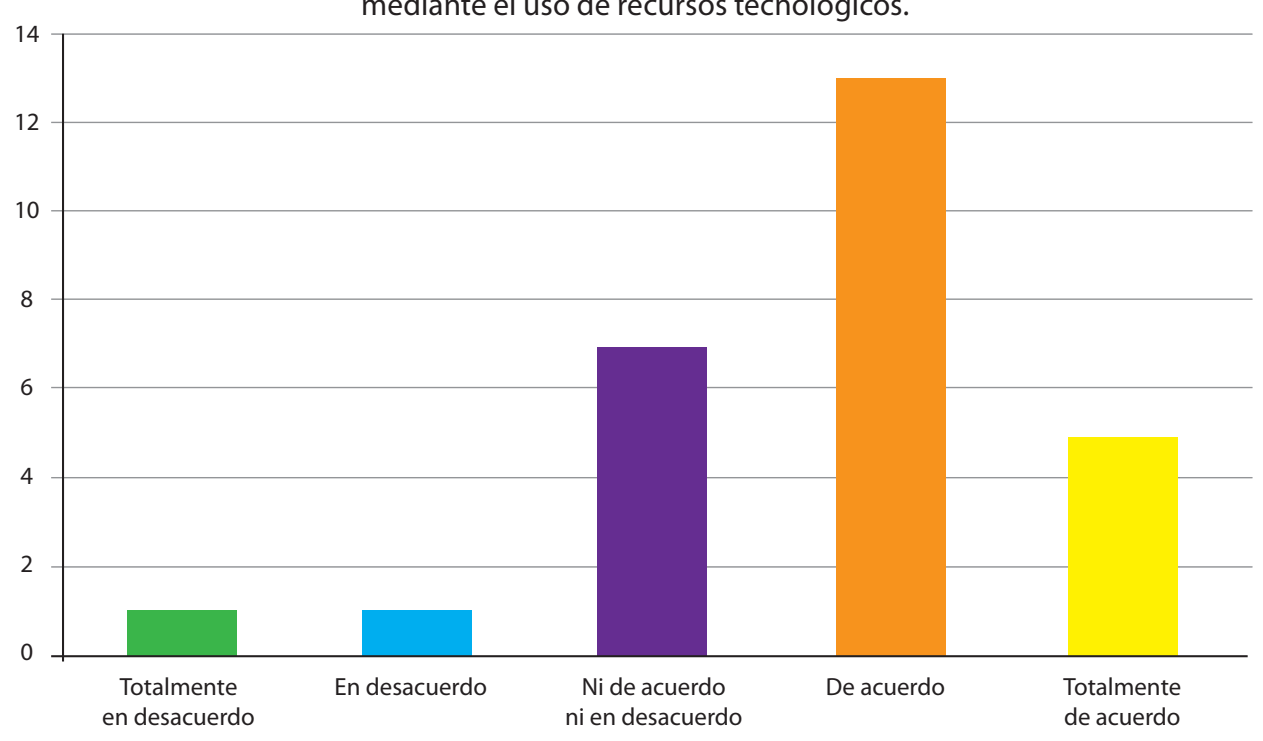

Fuente: elaboración propia.

Para efectos de vincular los recursos seleccionados por las personas docentes y sus posibilidades de brindar apoyo sustancial a los procesos de mediación pedagógica, también se les consultó sobre los criterios técnicos que consideraron o no para su elección.

Para el cumplimiento del objetivo número tres relacionado con el determinar por medio de grupos focales los fines educativos de los recursos y aplicaciones tecnológicas seleccionados, según su intencionalidad de creación. Se llevó a cabo un grupo focal con las ocho personas participantes. Ante la pregunta sobre: ¿cuáles son los recursos tecnológicos que usted utiliza en el desarrollo de los procesos de enseñanza-aprendizaje? Las ocho personas participantes indicaron que, utilizan computadora y celular para el desarrollo de sus lecciones con los estudiantes, lo mismo que las comunicaciones con padres y demás labores que deben realizar. Entre esas actividades, se mencionan los actos cívicos, las reuniones del personal docente. En términos generales, todas las personas coincidieron en las dificultades que tienen porque, muchos de los niños no cuentan con computadora y, mucho menos con Tablet, pero en todas las casas hay al menos un celular, razón que lo convierte en el medio de comunicación que tiene con los niños.

Con respecto a ¿cuáles son las aplicaciones tecnológicas que usted utiliza en el desarrollo de los procesos de enseñanza-aprendizaje? Las ocho personas participantes utilizan Microsoft Teams ${ }^{\circ}$ como aplicación tecnológica principal. En todos los casos, señalaron que esa aplicación es la que el MEP ha dispuesto para el desarrollo de comunicaciones con padres, estudiantes, lo mismo que para impartir las lecciones. Por lo tanto, su uso se justifica en primera instancia por ser de exigencia oficial. 
Los participantes números uno, dos, cuatro y cinco utilizan también WhatsApp', a pesar de que dicha aplicación no es oficial. Las personas participantes en el grupo focal comentaron que se les ha indicado que por parte de las autoridades del MEP, no debería ser parte de las herramientas a considerar. Aunado a lo anterior, las dos personas que son docentes de niños de primer grado indicaron que esta aplicación les permite una comunicación ágil y rápida con los padres de familia; pues los niños no son los que manejan Microsoft Teams. En esta misma línea, mencionaron que el tema de usar el correo electrónico también trae consigo la capacitación de los padres de familia para poder ingresar y, resulta ser más oportuno con el estudiantado de segundo ciclo.

Cuando se les preguntó ¿si saben cuál es la utilidad en el ámbito educativo que se da a los recursos tecnológicos? Todas las personas participantes en el grupo focal, atendieron el tema del uso que ellos le han otorgado y la efectividad que ven en la utilización de los recursos. Se debe indicar que, de manera puntual, ninguna de las personas manifestó de forma directa a las utilidades puntuales de los recursos.

En términos generales, el personal docente que participó en la actividad señaló que tanto la computadora como el celular se utilizan para brindar información y remitir material. Para todos los participantes, el celular es el medio más efectivo para remitir instrucciones y material a los niños, esto por cuanto muchos de los niños no cuentan con computadora. Al respecto, la participante número uno comentó que tiene a cargo 26 niños, de los cuales solamente tres tienen computadora.

Por otra parte, la persona participante número cuatro agregó que, si bien es cierto que el celular es el medio más adecuado y efectivo de comunicación y de envío de información, es necesario tener en cuenta que muchos de los padres lo que tienen es un solo celular y varios hijos; por lo tanto, se debe compartir el teléfono para que todos lo puedan utilizar. Finalmente, los participantes coincidieron en que, al utilizar el celular como medio de comunicación, resulta ser más práctico y efectivo para ellos, el proceso de la recolección de evidencias del trabajo de la población estudiantil.

Por otra parte, en cuanto a las aplicaciones más efectivas, para el grupo en general hay tres de ellas que son las que cumplen el criterio: a) WhatsApp', b) Microsoft Teams y c) correo electrónico.

En cuanto al WhatsApp ${ }^{\circ}$, consideraron que permite una comunicación rápida, efectiva con los padres, se puede enviar y recibir información. Si fuera el caso, sirve para aclarar consultas. Además, han descubierto que tiene una función por medio de la cual solo se mandan mensajes, pero no se pueden recibir, con esto han filtrado las comunicaciones innecesarias en el grupo. De esta manera, los padres que tengan alguna duda deben hacerlo de forma individual, con ello se permite una mejor atención de sus consultas.

En el caso de Microsoft Teams ${ }^{\circ}$ en primera instancia se utiliza porque es el medio oficial que les ha dado el MEP, de manera que hay una obligación de uso, pero resulta mejor para niños de segundo ciclo, por la madurez y atención que pueden tener.

Por otra parte, se contradice la información brindada en el grupo focal y, aquella que se obtiene de los cuestionarios y la escala de Likert, en cuanto a las aplicaciones más utilizadas y los usos que le dan. Según se determina por sus opiniones, el WhatsApps es la aplicación a la que tienen mayor acceso para comunicarse con sus estudiantes. En segundo lugar de uso está Microsoft Teams`, el cual es la herramienta oficial del MEP.

En concordancia con lo indicado por Cordero, De Roux, Jiany; Wong; Cattafi (2018) existe un esfuerzo por parte del personal docente, para favorecer la utilización tanto de los recursos como de las aplicaciones tecnológicas en los procesos formativos. Sin embargo, las situaciones tan complejas que comentan sobre las condiciones socioeconómicas de la población estudiantil que tienen a cargo, denota que usar las otras aplicaciones sería muy poco efectiva. Se interpreta que, el personal docente dio respuestas a partir de sus criterios y uso personal, no así de sus experiencias con la población estudiantil. Aunado a lo anterior, el personal docente requiere de espacios formales de formación en el uso de competencias digitales, para desarrollar procesos de mediación pedagógica utilizando recursos tecnológicos. 


\section{SÍNTESIS Y REFLEXIONES FINALES}

Como producto de los resultados obtenidos en la investigación y basado en el análisis de la información, se destacan las siguientes conclusiones:

Para Smith. (s.f.), el profesor debe estar capacitado en el uso de las herramientas digitales, conocer los procesos técnicos y didácticos que se requieren para hacer uso de ellas, con el fin de orientar al estudiante en su uso. En esta investigación, se concluye que la mayoría de las personas docentes participantes no pertenecen a la llamada era digital; es decir, no han tenido un gran contacto con el uso de las tecnologías; esto debido a la falta de capacitación por parte del MEP. Aun así, se reconoce el esfuerzo que están realizando por adaptarse a los cambios que implica el uso de la tecnología para el desarrollo de la mediación pedagógica.

También se encontró que el medio tecnológico más utilizado es el celular. Según fue referido por el equipo docente participante, es el medio más eficiente para tener un mayor contacto con los padres de familia. Además, reconocieron que es un recurso con el que la mayoría de los estudiantes cuenta. El segundo medio tecnológico que predomina en uso por parte del personal docente es la computadora.

Según se denota en los resultados de este trabajo, para todo el personal docente es fundamental que los medios utilizados cuenten con sistema de audio, micrófono y cámara. De esta manera, se favorecen los espacios de comunicación sincrónica y asincrónica.

Por otra parte, las aplicaciones tecnológicas que más utiliza el personal docente en el proceso educativo y, por ende, para la mediación pedagógica son Microsoft Teams ${ }^{\circ}$ y WhatsApp ${ }^{\circ}$. El criterio de elección que predomina para la elección de la aplicación tecnológica denominada WhatsApp', es la facilidad de uso por parte del estudiantado y las personas encargadas de los niños y las niñas. Esto por cuanto es un medio muy común de comunicación en Costa Rica y a nivel mundial. Además, es la aplicación que utilizan el cuerpo docente de primer ciclo.

Microsoft Teams es la aplicación tecnológica utilizada por ser aquella de carácter oficial, según las instrucciones del MEP. Sin embargo, el personal docente participante indicó que se utiliza en su mayoría con estudiantes de segundo ciclo, no para los de primer ciclo que les cuesta más y, se distraen con facilidad. A pesar de ello, el uso es limitado, dado que la población estudiantil con la que trabajan tiene limitantes para el uso de las aplicaciones y recursos tecnológicos, lo cual dificulta la conectividad por parte de todos los estudiantes.

Llama la atención el hecho de que esta aplicación promueve la interacción y comunicación sincrónica, pero según fue referido por las personas participantes, existen condiciones socioeconómicas y de competencias digitales en la población estudiantil y las personas responsables de la niñez que limitan el desarrollo de un conjunto de funciones que tiene la aplicación.

Otro de los criterios considerados por el personal docente para la elección de los medios y aplicaciones tecnológicas, fue la facilidad que tenían para comunicar las instrucciones del trabajo a desarrollar por parte del estudiantado de forma clara y precisa. Este tipo de actividad es fundamental para los docentes participantes; pues según refirieron, el logro de las diversas actividades y tareas escolares del estudiantado depende de ello. Se evidencia la tendencia y el predominio de intentar buscar el desarrollo de estrategias didácticas presenciales desde la virtualidad.

Los resultados evidencian que los docentes no han explorado lo suficiente y para fines educativos las aplicaciones Microsoft Teams ${ }^{\circ}$ y WhatsApp ${ }^{\circ}$; se evidencia que puede haber temor por incursionar en su uso, o bien porque no se les ha brindado las capacitaciones que realmente necesitan para conocer mejor el funcionamiento de estas. Por lo tanto, se concluye también que se encuentran en un proceso de 
aprendizaje sobre el uso de las aplicaciones tecnológicas antes mencionadas como uso educativo para la estrategia "Aprendo en casa", implementado por el MEP.

Se concluye que otras aplicaciones tecnológicas que podrían ser utilizadas para el desarrollo de procesos de mediación pedagógica como es el caso de Zoom o Facebook', son poco conocidas por el personal docente participante en el estudio.

Otro aspecto que se obtiene como resultado concluyente de esta investigación es que, los docentes han dado su mayor esfuerzo por aprender cada día más sobre el uso, tanto de las aplicaciones como de los recursos tecnológicos para lograr tener un mayor acercamiento con los estudiantes y los padres de familia.

Existe una gran limitante de conocimiento en el uso de aplicaciones y recursos tecnológicos por parte de los padres de familia, lo cual dificulta el proceso de educación a distancia para los estudiantes; pues son estos los encargados de este proceso.

El cuerpo docente participante en la investigación muestra gran preocupación por falta de recursos tecnológicos en familias numerosas; pues esto dificulta el proceso de enseñanza aprendizaje, ya que un celular lo utilizan hasta por cuatro estudiantes.

Se evidencia que el personal docente se siente angustiado y en general, denotaron también preocupación, por la falta de conectividad que tienen sus estudiantes. En cuanto a esta situación, fueron claros en referir que algunos poseen conectividad limitada o es prepago, lo cual les afecta cuando se dan clases por medio de la plataforma oficial o la descarga de algún trabajo enviado.

En cuanto a las limitaciones presentadas en el proceso de desarrollo de la investigación, se destaca que es un estudio que no permite generalizaciones de los resultados, dado que se llevó a cabo en una institución educativa solamente. Además, se trabajó con una selección de la población y quedaron por fuera las personas docentes de asignaturas que no están entre las básicas.

Finalmente, a partir de la investigación se recomienda el desarrollo de otros estudios bajo esta misma línea, pero que permitan la generalización de resultados que puedan ser aplicados, en busca de mejoramiento en cuanto al uso de recursos y aplicaciones tecnológicas para la mediación pedagógica a nivel de primaria. Otro estudio importante que se recomienda, es la determinación de las competencias digitales del personal docente a nivel de primaria, con ello se pueden preparar capacitaciones que atiendan carencias puntuales. 


\section{REFERENCIAS}

Acuña, M. (2017). WhatsApp como herramienta educativa. Obtenido de https://www.evirtualplus. com/whatsapp-como-herramienta-educativa/

Asociación educación abierta (2018). Los recursos tecnológicos evolucionan a tal ritmo que el docente se halla en un proceso de constante formación. Obtenido de https:/educacionabierta.org/ los-recursos-tecnologicos-evolucionan-a-tal-ritmo-que-el-docente-se-halla-en-un-proceso-deconstante-formacion/

Cordero, J.; De Roux, M.; Jiany, R.; Wong, L.; Cattafi, R. (2018). Recursos didácticos aplicados a la educación. En: Revista Investigación, pensamiento crítico. Vol. 6, No. 3, septiembre-diciembre, pp. 36-46.

Distancia, U. I. (2019). Estrategias para la enseñanza a través de Zoom. Obtenido de http://www. ucmconecta.edu.co/wp-content/uploads/2020/03/3.-Guia-estrategias-para-la-enseanza-a-travs-de-la-plataforma-zoom-profesores.pdf

García, C.; López de Ayala, M.; López, A.; Garcíá, C.(2014): “Los riesgos de los adolescentes en Internet: los menores como actores y víctimas de los peligros de Internet". Revista Latina de Comunicación Social, 69, pp. 462 a 485. http://www.revistalatinacs.org/069/paper/1020_UR/23es.html DOI: 10.4185/RLCS-2014-1020.

Hernández, C; Gómez, D. y Bladeras, M. (2014). Tecnologías de la información y la comunicación. En: Revista Electrónica Actualidades Investigativas en Educación.Volumen 14, Número 3 Setiembre Diciembre pp. 1-19 ISSN 1409-4703. Obtenida de: https://revistas.uma.es/index.php/innoeduca/ article/view/4956/5031

Martínez-Garrido, C. (2018). Utilidad de los recursos tecnológicos en educación. En: Innoeduca. International Journal of Technology and Educational Innovation. Vol. 4. No. 2. Diciembre 2018pp. 138-149 ISSN: 2444-2925 DOI: http://dx.doi.org/10.24310/innoeduca.2018.v4i2.4956

Mazas, C. R. (marzo de 2021). Qué es y para qué sirve Microsoft Teams. Recuperado de https://andro4all. com/guias/apps-android/que-es-y-para-que-sirve-microsoft-teams

Ministerio de Educación Pública (2019). Propuesta de Pruebas FARO. San José, Costa Rica: MEP.

Muñoz-Quezada, P.(2006). Educación y efectividad. En: Revista iberoamericana de educación, Obtenido de: https://www.researchgate.net/publication/28106768

Palmucci, M. (2015). El docente en la escena del aula y el uso de las TIC en su estrategia educativa. Recuperado de: http://fido.palermo.edu/servicios_dyc/publicacionesdc/vista/detalle_articulo. php?id_articulo=11010\&id_libro=535.

Programa del Estado de la Nación (2019). Informe del estado de la educación. Recuperado de: informe_estado_nacion_2019.pdf (estadonacion.or.cr)

Smith. (s.f.). El tesoro del saber tecnológico. Obtenido de https://sites.google.com/site/ eltesorodelsabertecnologico/el-papel-deldocente-en-la-era-digital

Soriano. (2020). Obtenido de https://theke.info/2020/09/28/las-competencias-digitales-en-el-ambitoeducativo/

Universidad Estatal a Distancia (2013). Glosario de términos curriculares. San José, Costa Rica: UNED. 